\title{
Agrotecnia 25
}

\author{
Edición especial
}

REBIOS 2017.

XI Reunión Nacional Científico-Técnica de Biología de SuelosCorrientes (Argentina)

\author{
Instituto Agrotécnico "Pedro M. Fuentes Godo" \\ Facultad de Ciencias Agrarias - UNNE
}




\title{
Comisión Organizadora REBIOS XI 2017
}

\author{
Presidente: \\ María IGLESIAS \\ Vice-Presidente: \\ Mónica COLLAVINO \\ Secretaría: \\ Marcela COSSOLI - Marina FORASTIER \\ Tesorería: \\ Amalia ROMERO - Andrea SIRIO \\ Edición: \\ Claudina HACK Ma. Elena CASTELAN \\ Miriam PORTA
}

\section{Organización General}

Luján ACOSTA
María José ADIS
Natalia AMBROGGIO
Silvia ARZUAGA
Oscar AYALA
Cristian BARRIONUEVO
Sebastian BLANCO
Angela Ma BURGOS
Sebastián CARNICER
Gustavo CASAFUS
Dolores COLOMBO
Amilcar CORREA
Eduardo FLACHSLAND
Melina FRESSONI

Roxana GARCÍA
Gonzalo GOMEZ
Graciela GOMEZ
Rosa GONZALEZ LEGUIZAMÓN
Yuliana GORSELOWSKI
Gabriela GUASCH
Facundo GUTIERREZ
Susana GUTIERREZ
Alexis HATSEMBILLER
Mariel KRINSKY
Corina LECONTE
María LOPEZ GASTÓN
Valeria MAMBRIN
Natalia MANSILLA
Alejandra MARTINA
Cecilia MARTIN
Laura NDURÉ

María PELLEGRINI GARCÍA

Germán PÉREZ

Sonia ROLDAN

Cristina SALGADO

Cristina SOTELO

Graciela TERADA

Marcela TOLEDO

Susana VASQUEZ

Katia ZACARIAS

Cátedra de Microbiología Agrícola (FCA-UNNE)

Cátedra de Edafología (FCA-UNNE)

Cátedra de Fitopatología (FCA-UNNE)

Cátedra de Conservación y Manejo de Suelo (FCA-UNNE)

Cátedra de Zoología Agrícola (FCA-UNNE)

Instituto Agrotécnico "Pedro M. Fuentes Godo" (FCA-UNNE) 


\section{Comisión científica}

$\begin{array}{ll}\text { Betina AGARAS } & \text { Susana GUTIERREZ } \\ \text { Ada ALBANESI } & \text { Enrique PIQUIN } \\ \text { Josefina AMIGO } & \text { Cecilia SANCHEZ } \\ \text { Oscar AYALA } & \text { Juan SILBERMAN } \\ \text { Silvia BENINTENDE } & \text { Alejandra STERREN } \\ \text { Mónica COLLAVINO } & \text { Tania TAURIAN } \\ \text { Olga CORREA } & \text { Elsa ULLA } \\ \text { Gabriela DI BARBARO } & \text { Luis WALL } \\ \text { Valeria FAGGIOLI } & \end{array}$

\section{Disertantes}

Dra.Betina AGARAS

Dr. Fernando Dini ANDREOTE

Ing. Agr. Oscar AYALA

Ing. Agr. Rafael BALIÑA

Lic. (Dr.) Camilo BEDANO

Ing.Agr. (Ms. Sc.) Agustín BIANCHINI

Ing. Agr. (Dr.) Marcelo CARMONA

Lic. (Dra.) Mónica COLLAVINO

Ing. Agr. Paulo D'ANDREA

Ing.Agr.(Dra.) Valeria FAGGIOLI

Lic. Sebastián FRACCHIA

Dra. Lilian FRIONI

Lic. (Dra.) Laura GASONI

Ing. Agr. (Dra.) Ana María GONZÁLEZ

Ing Agr. Gustavo GONZÁLEZ ANTA

Ing. Agr. (Dr.) Alejandro PERTICARI

Ing. Agr. (Dra.) Cristina SALGADO

Dra. Tania TAURIAN

Ing.Agr. (Ms. Sc.) Cecilia SANCHEZ

Ing.Agr. (Dr.) Juan SILBERMAN

Ing.Agr.(Dra.) Marcela TOLEDO

Lic. (Dr.) Luis WALL

Dr. Sergio ZALAZAR 


\title{
Comisión Permanente
}

\section{Reunión Nacional Científico Técnica de Biología de Suelos}

\author{
Ada ALBANESI (Microb. Agrícola FAyA -UNSE) \\ Josefina AMIGO (Microb. Agrícola FAZ-UNT) \\ Analía ANRIQUEZ (Microb. Agrícola FAyA -UNSE) \\ Silvia BENINTENDE (Microb. Agrícola FCA-UNER) \\ Marcelo BENITEZ AHRENDTS (Microb. Agrícola FCA-UNJu) \\ Carla BRUNO (FCA-UNRC) \\ Viviana CHIOCCHIO (Microb. Agrícola FA-UBA) \\ Mónica COLLAVINO (Microb. Agrícola FCA-UNNE) \\ Olga CORREA (Microb. Agrícola FA-UBA) \\ Gabriela DI BARBARO (Microb. Agrícola FCA-UNCA) \\ María IGLESIAS (Microb. Agrícola FcA-UNNE) \\ Susana KRIEGER (Microb. Agrícola FCA-UNSalta) \\ Enrique PIQUIN (Microb. Agrícola FCA-UNSalta) \\ Susana PERNASETTI (Microb. Agrícola FCA-UNCA) \\ Juan SILBERMAN (Microb. Agrícola FAyA -UNSE) \\ Alejandra STERREN (Microb. Agrícola FCA-UNER) \\ Alicia THUAR (FCA-UNRC) \\ Elsa ULLA (Microb. Agrícola FAZ-UNT)
}

